\title{
Aproximación terapéutica al uso y abuso de drogas recreativas
}

\author{
Miguel Angel Landabaso Vazouez*; Miguel Gutiérrez Fraile** \\ ${ }^{*}$ Centro de Drogodependencias Barakaldo, Vizcaya. Osakidetza-Servicio Vasco de Salud. \\ **Servicio de Psiquiatria, Hospital de Cruces Barakaldo, Vizcaya. Osakidetza; Catedra de Psiquiatria Universidad del País Vasco. \\ Enviar correspondencia: Miguel A Landabaso Vazquez. Centro de Drogodependencias Barakaldo. C/ Berriotxoa n² \\ 48901. Barakaldo Bizkaia.Tfno : 944388505. E-mail: miguelangelandabaso@ euskalnet.net.
}

\section{Resumen}

Objetivo: describir y analizar el estado actual del tratamiento del uso y abuso de las drogas recreativas y de sus efectos adversos de tipo psiquiátrico. Método: la información recopilada en la búsqueda bibliográfica se ha organizado por sustancias -MDMA, GHB (gammahidroxibutirato) y ketamina- y por el tipo de efectos. Resultados: a pesar de la escasez de publicaciones parece existir un cierto grado de acuerdo en el abordaje de los problemas con MDMA y GHB; para las complicaciones psiquiátricas agudas, subagudas y residuales por éxtasis se recomienda la utilización de benzodiazepinas, antidepresivos y/o antipsicóticos atípicos; para la intoxicación y síndrome de abstinencia producido por el GHB y similares, se recomienda la utilización de benzodiacepinas, y la utilización conjunta de estabilizadores del humor y antipsicóticos atípicos. En el caso del síndrome de abstinencia y de la sintomatología positiva, negativa y cognitiva que puede producir el consumo de Ketamina, ya que no existe un tratamiento protocolizado, se comentan resultados de recientes estudios. Conclusiones: dada la insuficiente experiencia clínica y terapéutica, así como la falta de estudios clínicos controlados, en la actualidad no existe un consenso terapéutico establecido para las drogas de recreo.

Palabras claves: Tratamiento farmacológico, MDMA, GHB, Ketamina.

\section{Summary}

Objective: to describe and analyze the state of the art of the treatment of use-abuse of "club drugs" and their psychiatric adverse effects. Method: data from literature were organized according to the type of drug -MDMA, GHB (Gammahidroxibutirate) and Ketamine- and to the type of adverse effects. Results: does exist certain agreements in how to approach problems related with the use of MDMA and GHB; for acute, subacute and residual psychiatric disorders induced by Ecstasy, the use of benzodiazepines, antidepressants and/or atypical antipsychotics has been suggested; for intoxication and withdrawal syndromes induced by GHB and similar drugs, the use of benzodiazepines, and the use of mood stabilizers and atypical antipsychotics has been suggested. With respect to withdrawal and positive, negative and cognitive simtomatology induced by Ketamine, and due to the fact that there is no treatment currently in use, the results reported by recent studies are discussed. Conclusion: due to the insuficient clinical and therapeutical experience and to the lack of controlled clinical trials, there is no therapeutic consensus for "club drugs".

Key Words: Pharmacologic treatment, MDMA, GHB, Ketamine. 


\section{INTRODUCCIÓN}

D ecía Claude Bernard "el gran principio experimental es la duda, la duda filosófica que deja al espiritu su libertad y su iniciativa, y de donde derivan las cualidades más preciadas para un investigador en fisiología y medicina"(1).

Es con esa duda con la que realizamos esta "aproximación", aproximación porque hablamos de sustancias "nuevas" en cuanto a su consumo y sobre todo en cuanto a su patología, que es lo que tenemos que tratar, terapéuticamente hablando. Terapeútica que está lejos de tener un consenso, protocolo o recomendación terapeútica que pueda ser avalada por estudios o experiencia clínica suficiente.

De cualquier forma vamos sabiendo un poco más de estas sustancias, y una vez más nos ocurre como hace unas décadas, en la que eran los pacientes (en aquel entonces heroinómanos) quienes nos "enseñaban" aproximaciones terapeúticas que hoy en día son compartidas y mejoradas por la mayoría de los que se dedican a esta "especialidad".

Desde hace unos años la popularidad de las denominadas drogas recreativas ("club drugs") ha ido en aumento; dentro de este grupo se describen diferentes sustancias con mayor o menor popularidad según la zona o país. Asi se describen dentro de este grupo el MDMA (éxtasis), Gammahidroxibutirato (GHB) y similares, Ketamina, Flunitracepán y LSD.

En nuetro país si tenemos cierta experiencia del abuso de Flunitracepán entre pacientes heroinómanos, asi como del tratamiento por consumo de LSD ${ }^{(2)}$; sin embargo existe escasa literatura y experiencia en el uso del Flunitracepán como droga recreativa.

Sin embargo parece ser que a todas ellas les unen sus efectos directos o indirectos sobre el sistema serotoninérgico y glutamatérgico ${ }^{(3,4,5,6)}$.

Puede ser que estas "modas o nuevos consumos" nos han pillado un poco "mayores" a los clínicos; dice una chirigota del carnaval de Cadiz "voy a colarme un sabadito de movida, en una fiesta pastillera por probar, pienso llevarme mis pastillas preferidas: una para la artrosis, el Almax y el Dolalgial"(7).

\section{MDMA (EXTASIS) y similares}

Desde la década de los 90 han ido apareciendo publicaciones con relación a las complicaciones psiquiátricas por uso o abuso de esta sustancia y similares.

Es difícil precisar si la sustancia actúa como desencadenante en personalidades premórbidas o induce alteraciones psiquiátricas en sujetos sanos; además hay que tener en cuenta que los niveles de MDMA en suero no se correlacionan directamente con los síntomas clínicos, aunque si parece ser que dosis elevadas se relacionan con episodios psicóticos inducidos ${ }^{(3)}$. Asi mismo hay que tener en cuenta que los consumidores habituales de MDMA no suelen ser "fieles" a esta sustancia, existiendo una tasa muy elevada de policonsumidores ${ }^{(8)}$.

En lo que sí parecen estar de acuerdo la mayoría de los investigadores es en la utilización preventiva de ISRS (fluoxetina y citalopran) al bloquear la liberación de serotonina inducida por $\operatorname{MDMA}^{(4,9,10)}$.

\section{- Aproximación terapéutica en las reac- ciones agudas psiquiátricas por MDMA (Intoxicaciones)}

Entre las reacciones agudas, que se presentan durante el consumo o en las 24 horas posteriores, se encuentran crisis de ansiedad, ataques de pánico, insomnio y "flashbacks" y en consumos altos (por encima de $200 \mathrm{mg}$ ) se ha descrito sintomatología paranoide y alucinaciones visuales y auditivas. En los que predominaria sintomatología orgánica grave (hipertermia, rabdomiolisis,...)

En estos casos se recomienda el tratamiento hospitalario con Diazepan, Loracepan - Midazolan para combatir la agitación junto a los tratamientos de urgencia apropiados para 
la sintomatología física acompañante (taquicardia, hipertermia, rabdomiolisis,...). En estos casos agudos, con patología orgánica asociada, hay que tener mucha precaución en la utilización de antipsicóticos e ISRS, ya que pueden agravar la hipertermia.

\section{- Aproximación terapéutica en las reac- ciones subagudas psiquiátricas por MDMA}

Estas reacciones se presentan a lo largo de las 2 semanas posteriores a la ingesta y no estarían relacionadas con la presencia de la sustancia en el organismo.

Se han descrito reacciones de somnolencia, astenia, síntomas depresivos, de ansiedad, repetición de ataques de pánico iniciados en la intoxicación e irritabilidad.

En estos casos se recomienda el tratamiento con Benzodiacepinas, con estricto control y durante el menor tiempo posible, por su capacidad de ser fármacos de abuso y capacidad de provocar dependencia. En los casos que exista sintomatología depresiva y/o irritabilidad se recomienda el tratamiento con ISRS (fluoxetina o citalopran) solo o en combinación con Mirtazapina, esta última también puede utilizarse como alternativa a las Benzodiazepinas por su capacidad inductora del sueño y relajante.

En el tratamiento con ISRS y/o Mirtazapina se recomienda el mantenimiento del tratamiento durante 6 meses o hasta la desaparición de la sintomatología.

\section{- Aproximación terapéutica a las reaccio- nes psiquiátricas crónicas o residuales por MDMA}

Este tipo de reacciones se presentan pasadas las 2 semanas de la última ingesta, donde la causalidad es muy difícil de valorar. Es en este tipo de patologías residuales donde residiría la discusión de si el MDMA actua como desencadenante en personalidades vulnerables.
En este tipo de reacciones o patologías residuales se incluyen alteraciones de memoria, trastornos depresivos mayores y cuadros psicóticos.

Según nuestra pequeña experiencia este grupo de pacientes estaría más relacionado con la gravedad de la sintomatología expresa$\mathrm{da}$, a diferencia de las reacciones subagudas en que la sintomatología parece ser de menor entidad.

También hay que tener en cuenta que los primeros trastornos psicóticos asociados al consumo de MDMA se remontan al año 1986; años en que los brotes psicóticos eran tratados con antipsicóticos clásicos y que la propia literatura definía como poco exitosos (8). En nuestra pequeña experiencia (11) los primeros pacientes habían sido tratados, sin resultado, con antipsicóticos clásicos durante un tiempo previo de 4 a 8 semanas. Es posible que en el momento actual, y dada la utilidad y eficacia de los antipsicóticos atípicos desde el inicio del brote psicótico, este tipo de pacientes pudieran ser integrados en el grupo de reacciones subagudas tras consumo de MDMA. Quedaría la discusión de los posibles efectos neurotóxicos de estas sustancias y su posible recuperación o no, para seguir integrando dentro de este grupo a los pacientes con patologías residuales ( alteraciones de memoria, síntomas parkinsonianos,,..$)$.

En los casos de sintomatología psicótica se recomienda el tratamiento con antipsicóticos atípicos. En nuestra experiencia Olanzapina a dosis de $10-15 \mathrm{mg} / 24 \mathrm{~h}$ el primer mes y $5-10 \mathrm{mg} / 24 \mathrm{~h}$ los siguientes meses con reducción gradual hasta la desaparición de la sintomatología. Si aparecen asociados síntomas afectivos recomendamos la asociación de antipsicóticos atípico con ISRS.

En los casos en que aparezcan trastornos afectivos mayores, se recomienda el tratamiento con ISRS o Velanfaxina a las dosis habituales para el tratamiento de un episodio depresivo mayor. Nosotros recomendamos, y esto sólo es una recomendación, no algo validado por la literatura, el uso conjunto de antipsicóticos atípico, a dosis menores de las 
habituales (2'5-5mg/24h en el caso de Olanzapina) junto a ISRS.

\section{GHB (GAMMAHIDROXIBUTIRATO) Y SIMI- LARES}

Con estas sustancias nos encontramos con dificultades añadidas (vease su evolución historica en capitulos precedentes); una de las mayores dificultades estriba en su rápida eliminación, no es posible detectarla en sangre más allá de las 2-8 horas y de las 8-12h en orina; incluso en pacientes con la función hepática comprometida parece ser que es completamente eliminada entre las 4-6-horas postingesta. Es decir es difícil unir sintomatología con determinación de consumo, lo que puede dificultar el diagnóstico.

Parece ser que el GHB y similares han sido usados para incrementar las sensaciones de euforia, relajación y sexualidad. Asi mismo se ha descrito que los efectos adversos aparecen más frecuentemente en policonsumidores que en los consumidores ocasionales de $\mathrm{GHB}$, como parece ser ocurre con la mayoría de las drogas "recreativas" y con las "¿no recreativas?".

Los usuarios describen la intoxicación por GHB como muy similar a la intoxicación por alcohol (recordad el uso del GHB para tratar el síndrome de abstinencia de alcohol)(3,12,13) y ambas sustancias pueden actuar sinérgicamente aumentando el riesgo de intoxicación y sobredosis.

Dado que la intoxicación por GHB y por alcohol son muy similares, los tratamientos protocolizados para la intoxicación de alcohol pueden ser validos para tratar la intoxicación por GHB.

Para el GHB y derivados se ha descrito un síndrome de abstinencia con sintomatología de insomnio, temblor, ansiedad, confusión, alucinaciones, delirium y taquicardia según la gravedad del mismo ${ }^{(3,5,14)}$.

Según diferentes autores, el tratamiento de primera elección en el síndrome de absti- nencia de GHB son las benzodiazepinas (Loracepan y Trazodona) a dosis similares a las utilizadas para provocar el sueño; asi mismo recomiendan la utilización conjunta de estabilizadores del humor (Gabapentina) y dosis bajas de antipsicóticos atípicos.

Aunque, de momento, no existe un tratamiento estandar protocolizado, si se recomienda la hospitalización y monitorización para el tratamiemnto con Benzodiacepinas a altas dosis en el caso de agitación y síntomas psicóticos.

\section{KETAMINA}

La Ketamina como su congenere PCP (fenilciclidina) son antagonistas de los receptores NMDA del sistema glutamatérgico y remedan sintomatología positiva, negativa y cognitiva que habitualmente se muestran en la esquizofrenia ${ }^{(15)}$.

Se ha hablado de un "síndrome de abstinencia" cuya sintomatología parece responder más al efecto del metabolito de eliminación retardada Norketamina (activo durante varios días) que al propio síndrome de abstinencia por falta de ketamina ${ }^{(16)}$.

Parece ser que los efectos y usos de esta sustancia tienen en común los efectos y usos de la cocaína, anfetaminas, opiáceos, alcohol y cannabis; con gran capacidad de producir, como en el caso de la cocaína, un alto deseo de consumo y una alta, rápida y duradera tolerancia hacia los efectos psicodélicos, como ocurre con los grandes consumidores de MDMA.

El patrón de uso compulsivo ("binges") se parece mucho al del consumo de cocaína y anfetaminas, por lo que el tratamiento aplicado a estos puede ser valido para el consumidor de ketamina.(17) Dado el mecanismo de acción de esta sustancia, los fármacos con actividad directa o indirecta sobre la regulación del receptor NMDA glutamatergico podrían ser validos; en el momento actual sabemos que los agentes con afinidad signifi- 
cativa por los receptores 5-HT2a serotoninérgicos antagonizan la hiperactividad que producen los antagonistas no competitivos del receptor NMDA glutamatérgico ${ }^{(18,19)}$.

Asi mismo se ha descrito el efecto positivo del piracetam para revertir los efectos negativos sobre la memoria producidos por la ketamina. Tambien la literatura refiere que dosis altas de vitamina $\mathrm{C}$ son efectivas en reducir el consumo compulsivo al intensificar la eliminación del metabolito activo Norketamina por la orina. Hay que reseñar un estudio en el que se dice que el pretratamiento con Nimodipino (antagonista de los canales $L$ del $\mathrm{Ca}++$ ) reduce la capacidad de la ketamina en producir sintomatología positiva, negativa y cognitiva ${ }^{(16,20)}$.

\section{CONCLUSIONES}

Dada la insuficiente experiencia clínica y terapéutica, así como la falta de estudios clínicos controlados, en la actualidad no existe un consenso terapéutico establecido para las drogas de recreo.

Todavía nos queda mucho por descubrir y comprender, y si estas nuevas "drogas de recreo" nos cogen con escasa experiencia terapéutica en ellas, también nos enseñan algo que en clínica vemos constantemente, que cada vez los/as pacientes tienden a mezclarse más (y la neurobiología de las drogas también), y ya no hay o casi no existen pacientes fieles a una sola sustancia.

Estas drogas recreativas nos muestran efectos mezclados de "drogas clásicas", pero, de momento, no tenemos tratamientos protocolizados, avalados, consensuados,... para ellas. La pregunta entonces es ¿en qué nos basamos? "en la experiencia de los expertos"; ¿y si no hay expertos todavía?: "podemos calcular aproximadamente un resultado sin necesidad de recurrir a pruebas matemáticas y tras una larga experiencia, los clínicos sabemos con una cierta aproximación que tipo de tratamiento le puede ir bien a un determinado paciente"(21).
Para terminar una cita de K.R.Popper ${ }^{(22)}$ que resume lo hasta aquí escrito, porque aunque no lo parezca, incluso en drogas, queremos hacer ciencia:" sea lo que sea que aceptemos, deberíamos cambiar solo como tanteo, siempre recordando que estamos, en el mejor de los casos, en posesión de una verdad parcial y que estamos destinados a cometer, al menos, alguna equivocación o error de juicio. En segundo lugar, deberíamos confiar, aunque fuera provisionalmente, en nuestra intuición solo si se ha llegado a ella como resultado de muchos intentos de utilizar nuestra imaginación, de muchos errores, de muchas pruebas, muchas dudas y de una búsqueda crítica".

\section{REFERENCIAS}

1. Bernard C. Introduction a l'etude de la medicine experimentale. Paris: Delagrange, 1934; pp: 115

2. Torrens Melich M, Ariño Viar J, Marina Gonzalez PA, Solé Puig J, Villa Canal A. Alucinógenos, cánnabis, benzodiazepinas, juego patológico. En: Fernández Miranda JJ, Gutierrez Cienfuegos E, Marina Gonzalez PA (eds). Actuaciones clínicas en trastornos adictivos. Madrid: Aula Médica, 2002.

3. Teter C, Guthrie SK. A comprehensive review of MDMA and GHB: two common club drugs. Pharmacotherapy 2001 ; 21: 1486-1513.

4. Malberg JE, Seiden LS. The role of ambient temperature and core temperature in amphetamine-induced neurotoxicity. En: Palomo T, Beninger R, Archer T (eds). Interactive monoaminergic disorders. Madrid: Ed Síntesis, 1999.

5. Miotto K, Darakjian J, Basch J, Murria S, Zogg MA, Rawson R.Gamma-hydroxybutyric acid: Patterns of use, effects and withdrawal. Am J Addict 2001; 10: 232-241.

6. Carpenter WT jr. The schizophrenia Ketamine challenge study debate. Biol Psychiatry 1999; 46: 1081-1091.

7. Los Alegres Divorciados, Chirigota de Cádiz Carnaval (CD). Voy de alegre divorciado. Motilla - Córdoba: Ed. Fonoruz, 2002. 
8. Bobes García J, González García-Portilla MP, Sáiz Martínez PA, González Seijo JC. Psicopatología y trastornos psiquiátricos relacionados con el uso de MDMA. En: Bobes García J, Lorenzo Fernández P, Sáiz Martínez PA (eds). Extasis (MDMA): Un abordaje comprehensivo. Tomo II. Barcelona: Masson, 1998.

9. Hekmatpanah CR, Peroutka SL. 5-hydroxytryptamine uptake blockers atenuate the 5hydroxytryptamine-releasing effect of 3,4methylenedioxymethamphetamine and related agents. Eur J Pharmacol 1990; 177: 95-98.

10. Liechti ME, Baumann C, Gamma A, Vollenweider FX. Acute psychological effects of 3,4methylenedioximethamphetamine (MDMA, "Ectasy") are attenuated by the serotonin uptake inhibitor citalopram. Neuropsychopharmacology 2000; 22: 513-521.

11. Landabaso MA, Iraurgi I, Jiménez-Lerma JM, Calle R, Sanz J, Gutiérrez-Fraile M. Ecstasyinduced psychotic disorder: Six-month followup study. Eur Addict Res 2002; 8 : 133-140.

12. Nimmerrichter AA, Walter H, Gutiérrez-Lobos KE, Lesch OM. Double-blind controlled trial of _-hydroxybutyrate and clomethiazole in the treatment of alcohol withdrawal. Alcohol Alcohol 2002; 1: 67-73.

13. Maremmani I, Lamanna F, Tagliamonte A. Longterm therapy using GHB (sodium gamma hydroxybutyrate) for treatment-resistant chronic alcoholics. J Psychoactive Drugs 2001; 33: 135-142.

14. McDaniel CH, Miotto KA. Gamma-hydroxybutirate $(\mathrm{GHB})$ and gamma -butyrolactone (GBL) withdrawal: Five case studies. J Psychoactive Drugs 2001; 32: 143-149.

15. Vollenweider FX, Vontobel P, Oye I, Hell D, Leenders KL. Effects of (S)-ketamine on striatal dopamine: A $(11 \mathrm{C})$ raclopride PET study of a model psychosis in humans. J Psychiatr Res 2000; 34: 35-43.

16. Jansen KLR, Darracot-Cankovic R. The nonmedical use of ketamine, part two: A review of problem use and dependence. J Psychoactive Drugs 2001; 33: 151-158.

17. Landabaso Vázquez MA, Casete Fernández L, Díaz González T, Sanz Etxeberría J, Terán Prieto A. Cocaína y otros psicoestimulantes. En: Fernández Miranda JJ, Gutiérrez Cienfuegos E, Marina Gonzalez PA (eds). Actuaciones clínicas en trastornos adictivos. Madrid: Aula Médica, 2002.

18. Callado LF, Meana J. Neurolépticos atípicos y consumo de sustancias. Bases farmacológicas. Trastornos Adictivos 2001; 3 (monográfico 2): 7-18.

19. Tollefson GD, Andersen SW. Should we consider mood disturbance in schizophrenia as an important determinant of quality of life?. J Clin Psychiatry 1999; 60: 23-28.

20. Krupitsky EM, Burakov AM, RomanovaTN, Grinenko NI, Grinenko AY, Fletcher J, Petrakis IL, Krystal JH. Attenuation of ketamine effects by nimodipine pretreatment in recovering ethanol dependent men: Psychopharmacologic implications of the interaction of NMDA and L-type calcium channel antagonists. Neuropsychopharmacology 2001; 25: 936-947.

21. Bousoño García M, García-Prieto A, González García-Portilla P, Sáiz Martínez PA, Bascarán Fernández MT, Bobes García J. De la psiquiatría basada en pruebas a la humilde clínica cotidiana. Una reflexión. En: Pichot P, Ezcurra J, González-Pinto A, Gutiérrez Fraile M (eds). Ciclo vital y trastornos psiquiátricos graves. Madrid: Aula Médica, 2002.

22. Popper KP. La sociedad abierta y sus enemigos II. Barcelona: Planeta-Agostini, 1992. 